\title{
Critical Heat Flux Prediction of Subcooled Pool Boiling Based on the Microlayer Model*
}

\author{
Yao-Hua ZHAO**, Takaharu TSURUTA*** \\ and Takashi MASUOKA****
}

\begin{abstract}
The critical heat flux (CHF) of subcooled boiling is theoretically predicted by means of the microlayer model. The enhancement of heat transfer for subcooled boiling is mainly due to the augmented heat convection caused by the forming and collapsing of individual bubbles. For a uniform heat flux surface, CHF approaches a constant in the high subcooling region. For a uniform temperature surface, CHF increases with the subcooling. The evaporative heat transfer becomes small and the total heat flux is mainly due to the heat conduction outside the evaporating area as the subcooling is increased. The boiling crisis (CHF) is caused by the local dryout of microlayer at low subcooling and by the rapid increase of the duration of bubble condensation with the increase of wall superheat at high subcooling.
\end{abstract}

Key Words : Critical Heat Flux, Subcooled Boiling, Pool Boiling, Microlayer Model, Boiling Heat Transfer

\section{Introduction}

Subcooled boiling, as a highly efficient method of heat transfer, has been used in practice. However, the mechanism of heat transfer has not been clarified even for pool boiling. In the past 40 years, some quasiempirical formulations have been proposed for the pool subcooled boiling heat transfer.

As reviewed by Elkassabgi and Lienhard ${ }^{(1)}$, the first correlation for critical heat flux (CHF) in subcooled pool boiling is that by Kutateladze $e^{(2)}$ based on the pre-heating hypothesis. The CHF is given by

$$
\frac{q_{\mathrm{CHF}}^{\mathrm{sub}}}{q_{\mathrm{CHF}}^{\mathrm{sat}}}=1+\text { const } \frac{\rho_{l} C_{p} \Delta T_{\mathrm{sub}}}{\rho_{v} h_{f g}},
$$

i.e., in addition to the heat flux at the CHF in saturated pool boiling, the CHF in subcooled boiling should include the part of heat flux to preheat the subcooled liquid to its boiling point.

* Received 12th February, 2002. Japanese original: Trans. Jpn. Soc. Mech. Eng., Vol.67, No. 654, B (2001), pp. 466-472 (Received 22nd December, 1999)

** Department of Mechanical Engineering, Kyushu Institute of Technology, Kitakyushu 804-8550, Japan/Institute of Engineering Thermophysics, Chinese Academy of Sciences, Beijing 100080, China. E-mail : yhzhao@mech.kyutech.ac.jp

*** Department of Mechanical Engineering, Kyushu Institute of Technology, Kitakyushu 804-8550, Japan

**** Department of Mechanical Engineering and Science, Kyushu University, Fukuoka 812-8581, Japan
Ivey and Morris ${ }^{(3)}$ modified Kutateladze's correlation by correlating the data of Kutateladze with their own data as follows.

$$
\frac{q_{\mathrm{CHF}}^{\text {sub }}}{q_{\mathrm{CHF}}^{\text {sut }}}=1+0.1\left(\frac{\rho_{g}}{\rho_{l}}\right)^{1 / 4} \frac{\rho_{l} c_{p} \Delta T_{\mathrm{sub}}}{\rho_{v} h_{f g}}
$$

Zuber et $\mathrm{al}^{(4)}$ proposed another correlation for $\mathrm{CHF}$ in subcooled pool boiling based on the so-called hydrodynamic instability model that has been used to predict the $\mathrm{CHF}$ in saturated pool boiling. Being different from saturated pool boiling, additional vapor, a condensed fraction of the departing vapor on the walls of jets through which it flowed, should be generated at a heater surface before the Helmholtz instability leads to burnout. The condensed vapor heat is determined by the transient heat conduction in the subcooled liquid during the lifetime of the vapor jet. The CHF correlation is given by

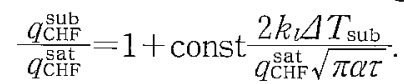

Here, the lifetime of the vapor jet $\tau$ is given by

$$
\tau=\frac{\pi \sqrt{2} \pi}{3}\left[\frac{\sigma}{g\left(\rho_{l}-\rho_{v}\right)}\right]^{1 / 2}\left[\frac{\rho_{v}{ }^{2}}{g\left(\rho_{l}-\rho_{v}\right) \sigma}\right]^{1 / 4} \text {. }
$$

From the above equation, $\tau$ gives values on the order of $1 \mathrm{~ms}$. However, Elkassabgi and Lienhard ${ }^{(1)}$ claimed that the motion pictures obtained in their experiment gave jet lifetimes on the order of $100 \mathrm{~ms}$ in the region of lower subcooling, and no vapor jet existed in the regions of moderate and high subcooling. Therefore the suitability of Eq. (3) is doubtful. 
Elkassabgi and Lienhard proposed different conceptual heat transfer models for subcooled pool boiling based on their experimental results. In their studies, three regions of subcooled burnout behavior were considered:

a) In the region of lowest subcooling, like Zuber's ${ }^{(4)}$ study, burnout occurs as a consequence of conventional hydrodynamic instability; however the $\mathrm{CHF}$ is augmented by the extra vapor that must be generated to satisfy condensation on the jet walls. The CHF, based on this model, is given by the following equation regressed from their own experimental data.

$$
\frac{q_{\mathrm{CH}}^{\mathrm{sub}}}{q_{\mathrm{CHF}}^{\mathrm{Sht}}}=1+4.28 \frac{\rho_{l} c_{p} \Delta T_{\mathrm{sub}}}{\rho_{v} h_{f g}}\left[\frac{\sigma^{3 / 4}}{\alpha\left[g\left(\rho_{l}-\rho_{v}\right)\right]^{1 / 4} \rho_{v}^{1 / 2}}\right]^{-1 / 4}
$$

b ) In the region of moderate subcooling, burnout occurs when the nucleate boiling process supplies heat to the saturated outer edge of the bubble layer faster than natural convection can remove it.

$$
N u=28+1.50 R a^{1 / 4} /\left(\beta \Delta T_{\mathrm{sub}}\right)^{7 / 8} \text {, }
$$

where the Nusselt number is defined as :

$$
N u \equiv q_{\mathrm{cHF}}^{\mathrm{sub}}\left(2 R_{\mathrm{eff}}\right) /\left(k_{i} \Delta T_{\mathrm{sub}}\right)
$$

and $R_{\text {eff }}$ is the sum of the radius of the heater wire and one departing bubble diameter. By using the Friz departing bubble diameter (for saturated pool boiling), $R_{\text {eff }}$ is obtained as

$$
R_{\text {eff }}=R_{\text {rod }}+0.02 \theta \sqrt{\sigma /\left[g\left(\rho_{l}-\rho_{v}\right)\right]} .
$$

c) In the region of high subcooling, burnout occurs when the nucleate boiling process itself is limited by the so-called molecular effusion and fails to provide energy as rapidly as it can be removed by natural convection. However, the experimental data are two orders of magnitude lower than the ideal limit by molecular effusion. The regressed correlation for $\mathrm{CHF}$ is

$$
q_{\mathrm{CH}}^{\mathrm{sub}}=\phi \cdot q_{\mathrm{mol}}, \phi=0.01+0.0047 \exp \left(-1.11 \times 10^{-6} \chi\right),
$$

where $\quad q_{\mathrm{mol}}=\rho_{\nu} h_{f g} \sqrt{R_{\mathrm{gas}} T_{\mathrm{sat}} / 2 \pi}$ and $\chi=R_{\mathrm{rod}}$ $\times \sqrt{R_{\text {gas }} T_{\text {sat }}} / \alpha$.

There is no doubt that there has been great progress in past studies on the subcooled pool boiling heat transfer. However, all of the above correlations are quasi-empirical ones, and their physical meanings and the $\mathrm{CHF}$ values predicted by them are totally different, especially in the higher subcooling region. Furthermore, in previous studies on subcooled pool boiling, the following problems remain:

1) The structure of liquid-solid contact is not considered. As a result, the details of heat transfer processes on and near the boiling surface (i.e., where and how the heat on the heater surface transfers to the boiling liquid) remain unclear.

2) The role of wall superheat in the CHF is not included in the previous studies. However, it is reasonable to consider the $\mathrm{CHF}$ as an inherent point on the continuous boiling curve and not as an isolated point.

3 ) The effects of properties of the heater surface on heat transfer should be considered to explain phenomena such as the CHF approaching a constant in some cases but increasing with the subcooling in others.

4) The main heat transfer type, the latent heat transfer, the convective heat transfer, or both, as well as their interaction should be quantitatively analyzed to explore the mechanism of heat transfer enhancement.

Recently, we have proposed a dynamic microlayer model ${ }^{(5)-(7)}$ that focuses on individual bubbles [Fig. 1] and can be used to explain the saturated pool boiling mechanism and to predict the heat flux in the nucleate boiling region at high heat flux and in the transition boiling region including the minimum heat flux point. In this model, the evaporation and partial dryout of the liquid microlayer underneath individual bubbles were considered to be important in nucleate boiling heat transfer, and the CHF has been derived as the maximum value of the possible heat flux. For transition boiling, in the low-superheat region, the heat flux is mainly due to the evaporation of the microlayer, and in the high-superheat region, the evaporations of the microlayer and the macrolayer [here, the macrolayer is different from that defined in the study of Haramura and $\left.\mathrm{Katto}^{(8)}\right]$ play important roles in heat transfer.

In the present study, the dynamic microlayer model is developed to explore the heat transfer mechanism and to predict the CHF for subcooled pool
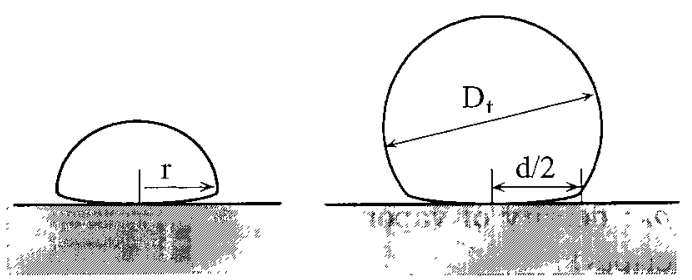

(a) The forming period (b) The evaporation period

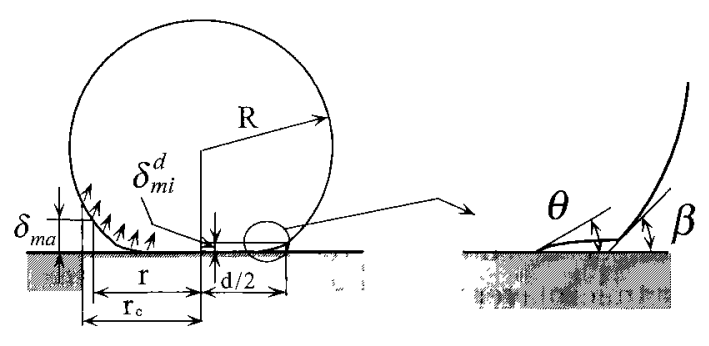

(c) Microlayer dryout and macrolayer

Fig. 1 Dynamic microlayer 
boiling.

\section{Nomenclature}

$A_{b}:$ microlayer area $=\pi(d / 2)^{2}$

$A_{d}$ : the largest cross-sectional area of an individual bubble

$A_{e v}$ : evaporating area of liquid layers

$A_{\text {ma }}$ : macrolayer area

$c_{p l}$ : specific heat of liquid

$D_{d}$ : departure diameter of an individual bubble

$D_{t}$ : instantaneous diameter of an individual bubble

$d$ : diameter of an individual bubble at the end of initial growth

$h_{f g}:$ latent heat of evaporation

$h_{v}$ : heat transfer coefficient of vapor condensation

$k_{l}:$ thermal conductivity of liquid

$N$ : density of active sites per unit area

$q$ : wall heat flux

$q_{e v}$ : evaporation heat flux on microlayer

$\bar{q}_{e v}^{\text {sat }}$ : mean evaporation heat flux in saturated pool boiling

$\boldsymbol{R}$ : radius of an individual bubble

$R_{\text {gas }}$ : gas constant

$r$ : coordination

$r_{c}$ : position at which the superheat boundary layer reaches the liquid-vapor interface

$r_{(t)}^{d}:$ radius of dryout area

$T:$ temperature

$T_{b}$ : bulk temperature of liquid

$t:$ time

$t_{g}:$ period of initial growth

$\alpha$ : thermal diffusivity of liquid

$\beta$ : configuration angle

$\Delta T_{\text {sat }}$ : wall superheat

$\Delta T_{\text {sub }}$ : liquid subcooling

$\delta_{\mathrm{ma}}:$ thickness of macrolayer

$\delta_{\mathrm{mi}}:$ thickness of microlayer

$\theta$ : contact angle

$\rho_{v}:$ density of vapor

subscripts :

$$
\begin{aligned}
& l: \text { liquid } \\
& v: \text { vapor } \\
& w: \text { heater surface }
\end{aligned}
$$

\section{Heat Transfer Model}

\subsection{Microlayer model for subcooled pool boiling heat transfer}

For subcooled boiling, the structures of the liquid layers (microlayer and macrolayer) are assumed to be the same as that of saturated pool boiling. Then, the local surface heat flux during the one period of an individual bubble can be given as follows:

$$
q_{(r, t)}= \begin{cases}0, & r \leq r_{(t)}^{d} \\ -\rho_{l} h_{f g} \frac{d \delta_{\mathrm{mi}}}{d t}, & r_{(t)}^{d}<r \leq d / 2 \\ -\rho_{l} h_{f g} \frac{d \delta_{\mathrm{ma}}}{d t}, & d / 2<r \leq r_{c} \\ q_{c}, & r \geq r_{c}\end{cases}
$$

where $r_{(t)}^{d}$ is the radius of dryout area. $\delta_{\mathrm{mi}}$ and $\delta_{\mathrm{ma}}$ are microlayer thickness and macrolayer thickness, respectively. The heat transfer in the dryout area has been neglected.

$\delta_{\mathrm{m}}$ and $r_{(t)}^{d}$ have been yielded in a previous study by the present authors ${ }^{(5)}$ and are given by:

$$
\begin{aligned}
& \delta_{\mathrm{mi}}=\frac{c \alpha \rho_{v} h_{f g} r}{2 k_{l} \Delta T_{s}} \cdot\left[1-\frac{8 c_{p l} k_{l}{ }^{2} \Delta T_{\mathrm{sat}}{ }^{3}\left(t-\tau_{g}\right)}{c^{2} \alpha h_{f g}{ }^{3} \rho_{v}{ }^{2} r^{2}}\right]^{1 / 2} \\
& r_{(t)}^{d}=\left[\frac{8 c_{p l} k_{l}{ }^{2} \Delta T_{\mathrm{sal}}{ }^{3}\left(t-\tau_{g}\right)}{c^{2} \alpha h_{f g}{ }^{3} \rho_{v}{ }^{2}}\right]^{1 / 2}
\end{aligned}
$$

Here, $\tau_{g}$ is the time at which the microlayer is formed at position $r$.

$$
\tau_{g}=c \alpha\left[\rho_{v} h_{f g} r /\left(2 k_{l} \Delta T_{\text {sat }}\right)\right]^{2}
$$

The conduction heat flux $q_{c}$ except the evaporated heat transfer consists of two parts: one is that in the macrolayer region, and the other is that in the microlayer region during the waiting time. The heat flux $q_{c}$ can be determined by transient heat conduction within the liquid layer until the superheated boundary layer reaches the liquid-vapor interface, i.e.,

$$
q_{c}=k_{l}\left(T_{w}-T_{0}\right) / \delta_{l},
$$

where $\delta_{l}$ is the equivalent thickness of the thermal boundary layer.

Therefore, the mean wall heat flux can be yielded from Eq. (9).

$$
\begin{aligned}
\bar{q}_{w} & =\frac{1}{A_{d} \tau_{d}}\left[\int_{0}^{\tau_{b}} \int_{A_{b}}\left(-\rho_{l} h_{f g} \frac{d \delta_{\mathrm{mi}}}{d t}\right) d A d t\right. \\
& +\int_{0}^{\tau_{b}} \int_{\pi r_{c}{ }^{2}-A_{b}}\left(-\rho_{l} h_{f g} \frac{d \delta_{\mathrm{ma}}}{d t}\right) d A d t \\
& \left.+\int_{0}^{\tau_{w}} \int_{A_{b}} q_{c} d A d t+\int_{0}^{\tau_{d}} \int_{A_{d}-A_{b}} q_{c} d A d t\right]
\end{aligned}
$$

Here, $\tau_{b}$ and $\tau_{d}$ are, respectively, the lifetime and the total period of an individual bubble, which will be derived in the next section.

The CHF is the maximum of the mean wall heat flux given by Eq. (14) and satisfies the following condition.

$$
\partial \bar{q}_{w} / \partial \Delta T_{\mathrm{sat}}=0, \text { as } \Delta T_{\mathrm{sat}}=\Delta T_{\mathrm{sat}}^{\mathrm{CHF}}
$$

\subsection{Behavior and period of individual bubble}

In the region of low subcooling, coalescence bubbles are formed. Their behaviors are considered to be the same as those in saturated pool boiling. However, the growth base size of coalescence bubbles decreases with the increase of liquid subcooling and is smaller than the most dangerous wavelength of Taylor instability, which is considered to be the growth base size of coalescence bubbles in saturated pool boiling.

In the region of high subcooling, many experiments have shown that the coalescence bubble is not 


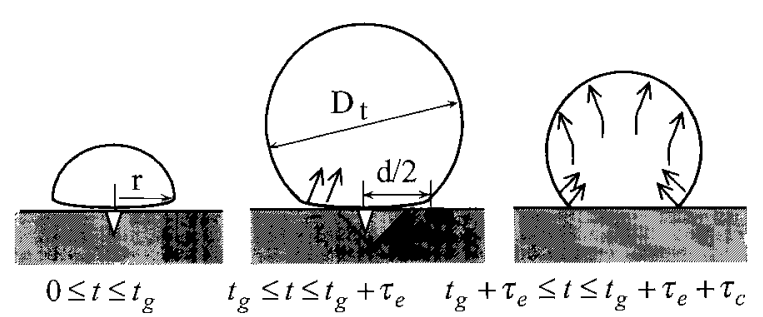

(a)

(b)

(c)

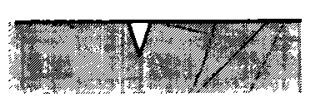

Waiting time $\tau_{w}$ (d)

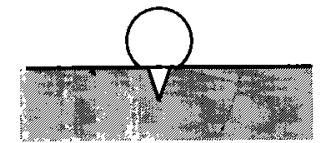

Activity of nuclei

(e)
Fig. 2 One cycle of individual bubble

substantially formed before burnout occurs. The heat transfer is mainly controlled by the behaviors of individual bubbles. Therefore, we only pay attention to the behaviors of individual bubbles at higher liquid subcooling in the present study.

One total period of an individual bubble consists of two parts, one is its lifetime, and the other is the waiting time of nucleus activity. The lifetime of the individual bubble consists of three durations, namely, the initial growth duration, the final growth duration and the condensation duration before the individual bubble collapses (Fig. 2).

2.2.1 The initial growth duration of the individual bubble $\left(0 \leq t \leq t_{g}\right) \quad$ During the initial growth of the individual bubbles, the semi-spherical bubbles grow from active nuclei and a microlayer is formed under the bubble. The growth equation of the individual bubbles and the initial thickness of the microlayer have been derived by the present authors ${ }^{(5)}$.

$$
\begin{aligned}
& R_{(t)} \equiv r=\frac{2 k_{l} \Delta T_{\text {sat }}}{\rho_{v} h_{f g} \sqrt{c \alpha}} t^{1 / 2}, 0 \leq t \leq t_{g} \\
& \delta_{\mathrm{mi}}^{0}=0.8 \sqrt{v_{t} t}=\sqrt{c \alpha \cdot t}=\frac{c \alpha \rho_{v} h_{\text {fg }} r}{k \iota \Delta T_{\mathrm{sat}}}, 0 \leq t \leq t_{g}
\end{aligned}
$$

where $c=0.64 \mathrm{Pr}$.

The initial growth duration of the individual bubble can be yield from Eq. (16) at $r=d / 2$,

$$
t_{g}=c \alpha\left[\rho_{v} h_{f g} d /\left(4 k_{l} \Delta T_{\mathrm{sat}}\right)\right]^{2} .
$$

2.2.2 The final growth duration due to evaporation of the microlayer $\left(t_{g} \leq t \leq t_{g}+\tau_{e}\right) \quad$ During the final growth period, the microlayer does not expand and the shape of bubble changes from semi-spherical to spherical segment geometry due to the evaporation of the microlayer. In this duration, a thicker liquid layer than the microlayer is formed under the bubble and among the adjacent individual. bubbles as shown in Fig. 1, which is termed a macrolayer by the present authors ${ }^{(5)}$. Meanwhile, vapor condensation occurs outside the evaporating area. In this duration, the energy equation can be given by

$$
\begin{aligned}
& 2 \pi \int q_{e v} r d r=\frac{d}{d t}\left[\frac{4 \pi}{3} \beta_{1} R^{3} \rho_{v} h_{f g}\right] \\
& \quad+2(1+\cos \beta) \pi R^{2} \dot{m} h_{f g}, t_{g} \leq t \leq t_{g}+\tau_{e} .
\end{aligned}
$$

Here, $\dot{m}$ is the condensation mass rate per unit area at the interface of bubbles. $\beta$ is the configuration angle of bubble as shown in Fig. $1 . \beta_{1}$ is given by

$$
\beta_{1}=\frac{1}{2}+\frac{3}{4} \cos \beta-\frac{1}{4} \cos ^{3} \beta \text {. }
$$

$\tau_{e}$ may be considered as the time at which the microlayer is completely dried out. It can be yield from Eq. (11) by using the following condition:

$$
\delta_{\operatorname{mi}(r=d / 2)}=0 \text {, at } t=t_{g}+\tau_{e},
$$

and $\tau_{e}$ is given by

$$
\tau_{e}=\frac{c^{2} \alpha h_{f g}{ }^{3} \rho_{v}{ }^{2} d^{2}}{32 c_{p l} k_{l}^{2} \Delta T_{s}^{3}} \text {. }
$$

Because $\tau_{e}$ is very short and the evaporation heat flux at the microlayer is very high, the effect of condensation heat is small compared to that of the evaporation heat and can be disregarded in this duration. Therefore, by considering the total heat balance, the bubble radius $R_{e}$ at the end of final growth can be derived from the following equations:

$$
\begin{aligned}
& \frac{2 \pi}{3}\left(\frac{d}{2}\right)^{2} \delta_{\mathrm{mi}(d / 2)}{ }^{0} \rho_{v} h_{f g}=\frac{4 \pi}{3} \beta_{1} R_{e}^{3} \rho_{v} h_{f g} \\
& R_{e}=\left[c \alpha \rho_{l} h_{f g} /\left(32 \beta_{1} k_{l} \Delta T_{\mathrm{sat}}\right)\right]^{1 / 3} \cdot d .
\end{aligned}
$$

2.2.3 The condensation duration $\tau_{c}\left(t_{g}+\tau_{e} \leq t \leq\right.$ $\left.t_{g}+\tau_{e}+\tau_{c}\right) \quad$ After the dryout of the microlayer, evaporation occurs mainly at the macrolayer surface. However, the evaporation heat flux is much smaller than that on the microlayer. On the other hand, the heat transfer by the vapor condensation on the interface of vapor bubble becomes dominant. The condensation process of bubble is controlled by the following equation until the bubble collapses.

$$
\begin{gathered}
\frac{d}{d t}\left[\frac{4 \pi}{3} \beta_{1} R^{3} \rho_{v} h_{f g}\right]=\int q_{e v} d A_{\mathrm{ma}} \\
-2(1+\cos \beta) \pi R^{2} h_{v} \Delta T_{\text {sub }}
\end{gathered}
$$

Initial condition : $R=R_{e}, t=t_{g}+\tau_{e}$

Bubble collapse condition : $R=0, t=t_{g}+\tau_{e}+\tau_{c}$

In Eq. (25), it has been considered that the heat transfer of vapor condensation is governed by the heat convection of subcooled liquid, i.e., $\dot{m} h_{f g}=h_{v} \Delta T_{\text {sub }}$. The heat transfer coefficient of vapor condensation $h_{v}$ obtained by Brucker and Sparrow ${ }^{(9)}$ is $h_{v}=10^{4} \mathrm{~W} / \mathrm{m}^{2} \mathrm{~K}$, which is independent of liquid subcooling. The evaporation heat transfer on the macrolayer can be given by the following equation that has been obtained by the present authors in their studies of transition boiling heat transfer ${ }^{(6),(7)}$.

$$
\begin{aligned}
& \int q_{e v} d A_{\mathrm{ma}}=\int \frac{k_{l} \Delta T_{\mathrm{sat}}}{\delta_{\mathrm{ma}}} d A_{\mathrm{ma}} \\
& \quad=2 \pi k_{l} \Delta T_{\mathrm{sat}} \sqrt{R^{2}-\left(\frac{d}{2}\right)^{2}}\left[1+\frac{2 c_{p} \Delta T_{\mathrm{sat}}}{\pi h_{f g}}\right]^{-1 / 2}
\end{aligned}
$$




$$
\begin{aligned}
& \cdot \ln \left[\sqrt{\frac{\pi h_{f g}}{2 c_{p} \Delta T_{\mathrm{sat}}}}\left(1+\sqrt{1+\frac{2 c_{p} \Delta T_{\mathrm{sat}}}{\pi h_{f g}}}\right)\right] \\
& -2 \pi k_{\imath} \Delta T_{\mathrm{sat}}\left[1+\frac{2 c_{p} \Delta T_{\mathrm{sat}}}{\pi h_{f g}}\right]^{-1} \sqrt{\pi \alpha t}
\end{aligned}
$$

As the wall superheat is small, $\pi h_{j g} \gg 2 c_{p} \Delta T_{\text {sat }}$, and the above equation can be approximately given by

$$
\int q_{e v} d A_{\mathrm{ma}} \cong \pi k_{l} \Delta T_{\mathrm{sat}} \sqrt{R^{2}-\left(\frac{d}{2}\right)^{2}} \cdot \ln \frac{\pi h_{f g}}{2 c_{p} \Delta T_{\mathrm{sat}}} .
$$

Thus, the lifetime of individual bubble $\tau_{1}$ is

$\tau_{1}=t_{g}+\tau_{e}+\tau_{c}$.

It is possible that the individual bubble departs from boiling surface before it collapses by vapor dynamics. If the departure time is represented by $\tau_{2}$, the attachment duration of bubble $\tau_{b}$ on the boiling surface is determined by

$$
\tau_{b}=\min \left[\tau_{1}, \tau_{2}\right] \text {. }
$$

For higher subcooling, it can be considered that $\tau_{b}$ is mainly dominated by the collapsing process because the attachment duration of bubble $\tau_{b}$ is short, i.e., $\tau_{b}=$ $\tau_{1}$.

2.2.4 The waiting time $\tau_{w}$ Immediately after the bubble collapse, the subcooled bulk liquid is supplied to the boiling wall. The thermal boundary layer grows by transient heat conduction in the subcooled liquid. The temperature profile in the boundary layer should be determined by solving the conjugated heat transfer in both the liquid and the heater wall. In the present study, we consider two special cases, which are the uniform temperature wall and the uniform heat flux wall.

(1) Uniform heat flux wall

For a thin heated surface with a heating rate $Q$ per unit area, the temperature distribution in the boundary layer is given ${ }^{(10)}$ by

$$
\begin{aligned}
& T_{L}-T_{b}=\frac{Q \alpha H}{k_{l}^{2}}\left[\frac{2 \mu}{\sqrt{\pi}} e^{-y^{2} / 4 \alpha t}-\operatorname{erfc} \frac{y}{2 \sqrt{\alpha t}}\right. \\
& \left.-\frac{k y}{\alpha H} \operatorname{erfc} \frac{y}{2 \sqrt{\alpha t}}+e^{\frac{k y}{\alpha H}+\mu^{2}} \operatorname{erfc}\left(\frac{y}{2 \sqrt{\alpha t}}+\mu\right)\right] .
\end{aligned}
$$

The wall heat flux $q$ is given by the following equation.

$$
q=Q\left(1-e^{\mu 2} \operatorname{erfc} \mu\right)
$$

Here, $\mu=(k / H) \sqrt{t / \alpha}, H=\rho_{s} C_{p s} \delta_{s}$.

In the present study, we consider the case in which the heat capacity of the heater wall $\rho_{s} C_{p s} \delta_{s}$ is very small, so the wall temperature $T_{w}$ and the wall heat flux $q$ can be approximately given by

$$
T_{w}-T_{b}=\frac{2 Q \sqrt{\alpha t}}{\sqrt{\pi} k_{l}}, q=Q .
$$

As shown in the above equations, the heater wall can be considered as a uniform heat flux wall, and the equivalent thickness of the thermal boundary layer is

$$
\hat{\delta}_{l}=2 \sqrt{a t} / \sqrt{\pi} \text {. }
$$

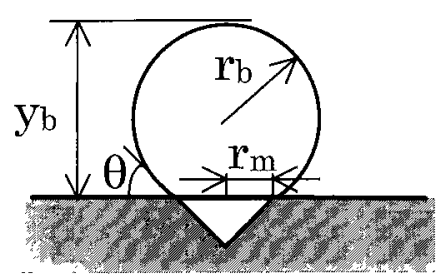

Vapor nuclei

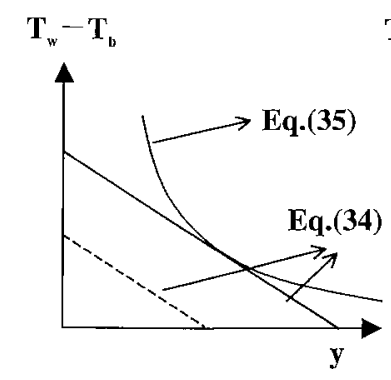

Uniform heat flux

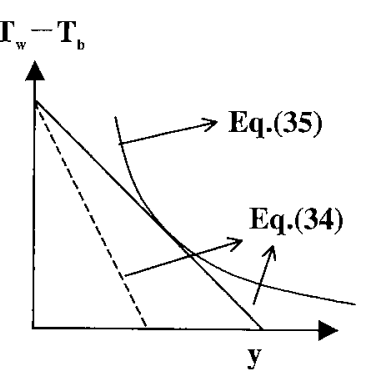

Uniform wall temperature
Fig. 3 Activity condition of vapor nuclei

Therefore, by using the equivalent thickness of the thermal boundary layer $\delta_{l}$ and the instantaneous wall temperature $T_{w}$, the temperature profile in the thermal boundary layer is linearly approached by

$$
T_{l}=T_{w}-\left(T_{w}-T_{b}\right) y / \delta_{l} \text {. }
$$

As is well known, in the thermal boundary layer, the activity condition for a nucleus site (cavity) with radius $r_{m}$ is given by

$$
\begin{aligned}
& T_{l}=T_{s}+2 \sin \theta \cdot \sigma T_{s} /\left(r_{m} h_{f g} \rho_{v}\right) \\
& \text { at } y=y_{b}=r_{b}(1+\cos \theta) .
\end{aligned}
$$

As shown in Fig. 3, the minimum waiting time can be yielded from Eqs. (34) and (35) as the temperature profile (Eq. (34)) becomes the tangent line of Eq. (35).

$$
\begin{aligned}
\tau_{w} & =\left[\frac{\pi(1+\cos \theta) \rho_{v} h_{f g} \gamma_{m}^{2}}{4 \sin ^{2} \theta \cdot \sigma T_{s}}\right] \frac{\left(T_{w}-T_{b}\right)^{2}}{\pi \alpha} \\
& =\left[\frac{\pi k_{l}}{2 Q}\right]^{2} \frac{\left(T_{w}-T_{b}\right)^{2}}{\pi \alpha},
\end{aligned}
$$

where $\theta$ is the contact angle. The radius of the activity cavity $\gamma_{m}$ is

$$
r_{m}=\left[\frac{2 \sigma T_{s} k_{l} \sin ^{2} \theta}{(1+\cos \theta) \rho_{v} h_{f g} Q}\right]^{1 / 2} .
$$

The instantaneous wall temperature $T_{w}$ satisfies the following equation.

$$
T_{w}-T_{s}=\left[\frac{8(1+\cos \theta) \sigma T_{s}}{\rho_{v} h_{f g} \delta_{l}}\right]^{1 / 2}\left(T_{w}-T_{b}\right)^{1 / 2}
$$

(2) Uniform temperature wall

For a uniform temperature wall, the equivalent thickness of the thermal boundary layer is

$$
\delta_{l}=\sqrt{\pi \alpha t},
$$

and the minimum waiting time is given by

$$
\tau_{w}=\left[\frac{8(1+\cos \theta) \dot{\sigma} T_{s}}{\rho_{v} h_{f g}}\right]^{2} \frac{\left(T_{w}-T_{b}\right)^{2}}{\pi \alpha\left(T_{w}-T_{s}\right)^{4}} .
$$

The total period of individual bubble is the sum of the all of the four durations: 


$$
\tau_{d}=\tau_{b}+\tau_{w}=t_{g}+\tau_{e}+\tau_{c}+\tau_{w}
$$

\section{3 CHF of subcooled pool boiling}

In the lower subcooling region, a coalescence bubble is formed similar to that in the saturated pool boiling. To consider the existence of the coalescence bubble, the mean initial temperature of fresh liquid in the macrolayer is assumed to be the average value of the wall temperature and the bulk liquid temperature during one period of coalescence bubble, i.e., $T_{0}=\left(T_{w}\right.$ $\left.+T_{b}\right) / 2$. Thus, the mean wall heat flux is given by the following equation. The $\mathrm{CHF}$ is obtained by the maximum value of this equation.

$$
\bar{q}_{w}=\bar{q}_{e v}^{\mathrm{sat}}+\left(1-\frac{\pi}{16}\right) \frac{k_{l}\left(\Delta T_{\mathrm{sat}}+\Delta T_{\mathrm{sub}}\right)}{\sqrt{\pi \alpha \tau_{d}}}
$$

where $q_{e v}^{\text {sat }}$ and $\tau_{d}$ are given by the same method as that in saturated pool boiling(5). The nuclei activity density is also assumed to be the same as that in saturated pool boiling, which is given ${ }^{(11)}$ by

$$
N=9.425 \times 10^{-6} \Delta T_{s}^{8.25} \text {. }
$$

The calculated results of the CHF are shown in Figs. 2 and 3, which are very close to the empirical relationship by Ivey and Morris ${ }^{(3)}$.

In the higher subcooling region, by neglecting the evaporation heat at the macrolayer, the mean evaporated heat flux is given by

$$
\bar{q}_{e v}=\frac{2 \pi}{3}\left(\frac{d}{2}\right)^{2} \delta_{\mathrm{mi}(d / 2)}^{0} \rho_{v} h_{f g} /\left[A_{d}\left(t_{g}+\tau_{e}^{\prime}+\tau_{c}+\tau_{w}\right)\right] .
$$

By adding the conducted heat flux, the total mean wall heat flux is obtained from the following equation.

$$
\begin{gathered}
\bar{q}_{w}=\bar{q}_{e v}+\frac{A_{\mathrm{mi}} \tau_{w}}{A_{d} \tau_{d}} \frac{2 k_{l}\left(\Delta T_{\mathrm{sat}}+\Delta T_{\mathrm{sub}}\right)}{\sqrt{\pi \alpha \tau_{w}}} \\
+\frac{A_{\mathrm{ma}}}{\bar{A}_{a}} \frac{2 k_{l}\left(\Delta T_{\mathrm{sat}}+\Delta T_{\mathrm{sub}}\right)}{\sqrt{\pi \alpha \tau_{d}}}
\end{gathered}
$$

In the above equations, for a uniform wall heat flux surface, the total period of the individual bubble $\tau_{c}$ is

$$
\begin{aligned}
\tau_{d} & =c \alpha\left[\frac{\rho_{v} h_{f g} d}{4 k_{l} \Delta T_{\mathrm{sat}}}\right]^{2}+\frac{c^{2} \alpha h_{f g}{ }^{3} \rho_{v}{ }^{2} d^{2}}{32 c_{p l} k_{l}^{2} \Delta T_{s}{ }^{3}} \\
& +\left[\frac{\pi k_{l}}{2 \bar{q}_{w}}\right]^{2} \frac{\left(T_{w}^{\prime}-T_{b}\right)^{2}}{\pi \alpha}+\tau_{c},
\end{aligned}
$$

and for a uniform wall temperature surface, $\tau_{d}$ is

$$
\begin{aligned}
\tau_{d} & =c \alpha\left[\frac{\rho_{v} h_{f g} d}{4 k_{l} \Delta T_{\mathrm{sat}}}\right]^{2}+\frac{c^{2} \alpha h_{f g}{ }^{3} \rho_{v}{ }^{2} d^{2}}{32 c_{p l} k_{l}^{2} \Delta T_{s}^{3}} \\
& +\left[\frac{8(1+\cos \theta) \sigma T_{s}}{\rho_{v} h_{f g}}\right]^{2} \frac{\left(T_{w}-T_{b}\right)^{2}}{\pi \alpha\left(T_{w}-T_{s}\right)^{4}}+\tau_{c} .
\end{aligned}
$$

In the case of $\theta=20^{\circ}$, the present $\mathrm{CHF}$ on the uniform wall heat flux surface is shown in Fig. 4. It is clear that the CHF approaches a constant as the subcooling increases. This phenomenon has been confirmed experimentally by some investigators ${ }^{(1),(12)}$. The mechanism can be considered as follows: the waiting time $\tau_{d}$ increases with the second power of liquid subcooling to dominate the total period of individual bubble compared to the other durations

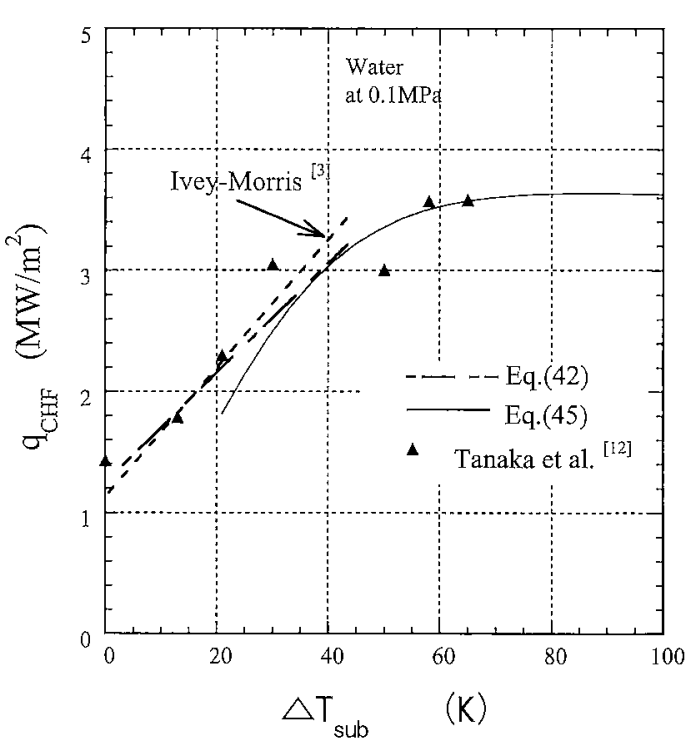

Fig. $4 \mathrm{CHF}$ vs. $\Delta T_{\text {sub }}$ for uniform heat flux surface

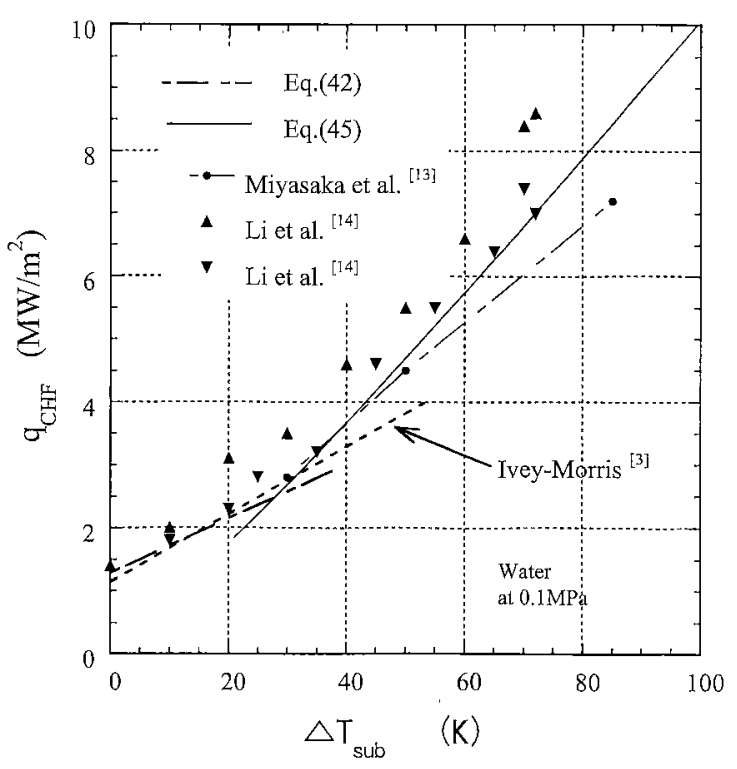

Fig. $5 \mathrm{CHF}$ vs. $\Delta T_{\text {sub }}$ for uniform temperature surface

(Eq. (36)) .

At the same contact angle of $\theta=20^{\circ}$, the present $\mathrm{CHF}$ for a uniform temperature surface is shown in Fig. 5. From Eq. (40), the waiting time is almost in inverse proportion to the fourth power of wall superheat and rapidly decreases with the increase of wall superheat. Therefore, the total period of the individual bubble is dominated by the condensation duration in the higher subcooling region. As a result, the CHF increases with the increase of liquid subcooling. A surface with good conductivity and large capacity may be approximately considered a uniform temperature surface. The experimental CHF results obtained by Miyasaka et al. ${ }^{(13)}$ and Li et al. ${ }^{(14)}$ (shown in Fig. 5) confirm the present predictions.

The occurrence mechanism of CHF, in the low 
subcooling region, is the same as that in saturated pool boiling, which is caused by the local dryout of microlayer. However, in the higher subcooling region, the evaporation heat transfer on the macrolayer becomes larger with the increase of wall superheat, and thus the bubble collapse duration becomes longer. Therefore, the maximum value of mean wall heat flux exists, which is the CHF.

For both the uniform temperature surface and the uniform heat flux surface, the present calculated results show that the evaporated heat transfer becomes smaller and the conducted heat transfer mainly at the macrolayer area becomes larger as the liquid subcooling increases. At high subcooling, the total heat transfer is almost fully contributed by the conducted heat transfer.

\section{Conclusion}

The critical heat flux (CHF) of subcooled boiling is theoretically predicted by using the microlayer model in this study.

The analysis shows that the enhancement of heat transfer for subcooled boiling is mainly due to the augmented heat removal caused by the forming and collapsing of individual bubbles. The heat transfer is strongly dependent on the total period of the individual bubble, which consists of the activity waiting time and the lifetime of the individual bubble. By formulating the behaviors of individual bubbles and the local heat flux of boiling surface, the $\mathrm{CHF}$ is predicted for two special cases, i.e., the uniform heat flux surface and the uniform temperature surface. For these two cases, the predicted CHF is close to the results of Ivey and Morris in the lower subcooling region. However, for a uniform heat flux surface, the CHF approaches a constant in the high subcooling region because the bubble activity waiting time becomes longer as subcooling becomes larger. On the other hand, for a uniform temperature surface, the CHF increases with the subcooling because the bubble activity waiting time is shorter. Furthermore, the evaporative heat transfer becomes very small and the total heat flux is mainly due to the heat conduction outside the evaporating area as the subcooling becomes large. The boiling crisis (CHF) is caused by the local dryout of microlayer at low subcooling and by the rapid increase of bubble condensation duration at high subcooling as the superheat approaches that of the CHF.

\section{References}

(1) Elkassabgi, Y. and Lienhard, J.H., Influences of Subcooling on Burnout of Horizontal Cylindrical Heaters, J. Heat Transfer, Vol.110 (1988), pp. $479-486$.

(2) Kutateladze, S.S., Hydrodynamic Theory of Changes in the Boiling Process Under Free Convection Conditions, Izv. Akad. Nauk. SSSR, Otd. Tekh. Nauk., No. 4 (1951), p. 529.

(3) Ivey, H.J. and Morris, D.J., Critical Heat Flux of Saturation and Subcooled Pool Boiling in Water at Atmospheric Pressure, Proc. 3rd Int. Heat Transfer Conf., Vol. III (1966), pp. 129-142.

(4) Zuber, N., Tribus, M. and Westwater, J.W., The Hydrodynamic Crisis in Pool Boiling of Saturated and Subcooled Liquids, International Developments in Heat Transfer, No. 27 (1963), pp. 20-236.

(5) Zhao, Y.H., Masuoka, T. and Tsuruta, T., Unified Theoretical Prediction of Fully Developed Nucleate Boiling and Critical Heat Flux Based on A Dynamic Microlayer Model, Int. J. Heat Mass Trans., Vol. 45 (2002), pp. 3189-3197.

(6) Zhao, Y.H., Masuoka, T. and Tsuruta, T., Prediction of Transition Boiling Heat Transfer Based on Partial Dryout Model of Liquid Layers, Trans. Jpn. Soc. Mech. Eng., (in Japanese), Vol.62, No. 599, B (1996), pp. 2717-2722.

(7) Zhao, Y.H., Masuoka, T. and Tsuruta, T., Theoretical Studies of Transient Pool Boiling Based on Micro/Macro-layer Model, Proc. of Convective Flow and Pool Boiling, Irsee, Germany, (1997).

(8) Haramura, Y. and Katto, Y., A New Hydrodynamic Model of Critical Heat Flux, Applicable Widely to Both Pool and Forced Convection Boiling on Submerged Bodies in Saturated Liquids, Int. J. Heat Mass Trans., Vol. 26 (1983), pp. 389-399.

(9) Brucker, G.G. and Sparrow, E.M., Direct Contact Condensation of Steam Bubbles in Water at High Pressure, Int. J. Heat Mass Transfer, Vol. 20 (1978), pp. 371-381.

(10) Lurie, H. and Johnson, H. A., Transient Pool Boiling of Water on a Vertical Surface with a Step in Heat Generation, J. Heat Transfer, Vol. 84 (1962), pp. 217-224.

(11) Gaertner, R.F., Photographic Study of Nucleate Pool Boiling on a Horizontal Surface, Trans. of ASME J. Heat Transfer, Vol. 87 (1965), pp. 17-29.

(12) Tanaka, H., Nishio, S. and Sugimoto, R., Bubble Structure in High-Heat-Flux Boiling, Proc. 36th National Heat Transfer Symposium of Japan, (in Japanese), (1999), pp. 607-608.

(13) Miyasaka, Y., Inada, S. and Owase, Y., Critical Heat Flux and Subcooled Nucleate Boiling in Transient Region between a Two-Dimensional Water Jet and a Heated Surface, J. Chemical Engineering of Japan, Vol. 13, No.1 (1980), pp. 29-35.

(14) Li, J., Yokoya, S., Watanabe, M., Yoshii, H. and Shoji, M., Study of Critical Heat Flux in Subcooled Pool Boiling, Proc. 36th National Heat Transfer Symposium of Japan, (in Japanese), (1999), pp. 391-392. 